\title{
Pandemic influenza A virus codon usage revisited: biases, adaptation and implications for vaccine strain development
}

\author{
Natalia Goñi ${ }^{1 \dagger}$, Andrés Iriarte ${ }^{2,3 \dagger}$, Victoria Comas ${ }^{1}$, Martín Soñora ${ }^{1}$, Pilar Moreno ${ }^{1,4}$, Gonzalo Moratorio ${ }^{1,5}$, \\ Héctor Musto ${ }^{2}$ and Juan Cristina ${ }^{1 *}$
}

\begin{abstract}
Background: Influenza A virus (IAV) is a member of the family Orthomyxoviridae and contains eight segments of a single-stranded RNA genome with negative polarity. The first influenza pandemic of this century was declared in April of 2009, with the emergence of a novel H1N1 IAV strain (H1N1pdm) in Mexico and USA. Understanding the extent and causes of biases in codon usage is essential to the understanding of viral evolution. A comprehensive study to investigate the effect of selection pressure imposed by the human host on the codon usage of an emerging, pandemic IAV strain and the trends in viral codon usage involved over the pandemic time period is much needed.
\end{abstract}

Results: We performed a comprehensive codon usage analysis of 310 IAV strains from the pandemic of 2009. Highly biased codon usage for Ala, Arg, Pro, Thr and Ser were found. Codon usage is strongly influenced by underlying biases in base composition. When correspondence analysis (COA) on relative synonymous codon usage (RSCU) is applied, the distribution of IAV ORFs in the plane defined by the first two major dimensional factors showed that different strains are located at different places, suggesting that IAV codon usage also reflects an evolutionary process.

Conclusions: A general association between codon usage bias, base composition and poor adaptation of the virus to the respective host tRNA pool, suggests that mutational pressure is the main force shaping H1N1 pdm IAV codon usage. A dynamic process is observed in the variation of codon usage of the strains enrolled in these studies. These results suggest a balance of mutational bias and natural selection, which allow the virus to explore and re-adapt its codon usage to different environments. Recoding of IAV taking into account codon bias, base composition and adaptation to host tRNA may provide important clues to develop new and appropriate vaccines.

Keywords: Influenza A virus, Codon usage, Evolution

\section{Background}

Influenza A virus (IAV) is a member of the family Orthomyxoviridae and contains eight segments of a singlestranded RNA genome with negative polarity [1]. IAV is one of the most important infectious diseases in humans [2]. Unlike most pathogens where exposure leads to lasting immunity in the host, IAV presents a moving antigenic

\footnotetext{
* Correspondence: cristina@cin.edu.uy

${ }^{\dagger}$ Equal contributors

'Laboratorio de Virología Molecular, Centro de Investigaciones Nucleares, Facultad de Ciencias, Universidad de la República, Iguá 4225, Montevideo 11400, Uruguay

Full list of author information is available at the end of the article
}

target [3], evading specific immunity triggered by previous infections. This process, called antigenic drift, is the result of the selective fixation of mutations in the gene encoding the hemagglutinin (HA) protein and to a lesser extent in the neuraminidase (NA) protein [4]. Variants that best escape the host immune response are thought to have a significant reproductive advantage [5]. Another process, called reassortment, is also considered a major force in the evolution of IAV [4]. It occurs when the virus acquires an HA and/or NA of a different IAV subtype (via reassortation) of one or more gene segments. This process has
C Biomed Central

(C) 2012 Goñi et al.; licensee BioMed Central Ltd. This is an Open Access article distributed under the terms of the Creative Commons Attribution License (http://creativecommons.org/licenses/by/2.0), which permits unrestricted use, distribution, and reproduction in any medium, provided the original work is properly cited. 
been in the basis of the devastating influenza pandemics that occurred several times in the last century [6].

The first influenza pandemic of this century was declared in April of 2009, with the emergence of a novel H1N1 IAV strain (H1N1pdm) in Mexico and USA $[7,8]$. By November of 2009, the virus was detected in about 207 countries, infecting more than 620,000 individuals worldwide and accounting for more than 7,800 deaths [7]. This strain was a multiple reassortant with genes derived from viruses that originally circulated in the swine, avian and human populations [9].

It has been observed that IAV is subjected to host immune selection pressure and undergoes rapid evolution, especially when the virus crosses the host species barrier [10]. The replication cycle of IAV depends on host machinery and the virus utilizes host cellular components for its protein synthesis. Therefore, the interplay of codon usage of virus and host could affect viral replication. For these reasons, a detailed understanding of IAV evolution and host adaptation is crucial.

Due to the degeneracy of the genetic code, most amino acids are coded by more than one codon. Synonymous triplets are not used randomly. In several organisms, natural selection and mutational input seem to bias codon use toward a certain subset of codons [11]. Two major models have been proposed to explain codon usage: the translational selection and the mutational models [12]. Codon usage bias related to translation efficiency (at two different levels: speed and accuracy) seems to be linked to local cognate isoacceptors tRNAs abundances, which in turn determine the major codon preferences [13]. On the other hand, discrepancies on codon usage could be due to genome compositional constraints and mutational biases [14]. Nevertheless, these two models cannot be considered as mutually exclusive.

Although previous studies have been performed on the general codon usage of IAV $[2,12,15,16]$, a deep and comprehensive study to investigate the effect of selection pressure imposed by the human host on the codon usage of an emerging, pandemic IAV strain and the trends in viral codon usage involved over the pandemic time period is much needed.

In order to gain insight into these matters, we performed a comprehensive codon usage analysis of 310 H1N1pdm IAV strains, isolated from April to September of 2009, for which the complete genome sequences are available.

\section{Results}

In order to study the extent of codon usage bias in H1N1pdm IAV strains in relation to seasonal H1N1 and H3N2 as well as human and swine host cells, the relative synonymous codon usage (RSCU) [14] values for each codon were calculated for the $310 \mathrm{H} 1 \mathrm{~N} 1 \mathrm{pdm}$ strains enrolled in these studies and compared with seasonal IAV strains and host organisms. The results of these studies are shown in Table 1.

All codons containing the dinucleotide $\mathrm{CpG}$ were underrepresented in all IAV viruses. Important differences were found between human and swine hosts and IAV strains. Particularly, high biased frequencies $(\triangle \mathrm{RSCU} \geq 0.30)$ were found for Leu, Ile, Val, Ser, Pro, Thr, Ala, His, Gln, Glu, Arg and Gly. Interestingly, the huge majority of preferred codons in the viruses are A-ended. In the case of Arg, there is a strong bias towards an increase in AGA and AGG, while the CGN codons are depleted (see Table 1).

To observe if H1N1pdm IAV strain sequences display similar codon usage biases, the effective number of codons (ENC) [17] values were calculated for the 310 strains enrolled in this study (mean of $52.51 \pm 0.05$ ). ENC varies from 20 to 61, where the larger the extent of codon bias in a gene, the smaller the ENC value. Thus, a value of 52.5 strongly suggests that the overall codon usage among these strains is only slightly biased.

Since codon usage by its very nature is multivariate, it is necessary to analyze the data using multivariate statistical techniques, like correspondence analysis (COA) [18]. The correlation between the position on the first dimensional factor generated by this analysis on RSCU (20.7\% of the total variability) for each strain and the respective $\mathrm{G}+\mathrm{C}$ content at synonymous variable third position $\left(\mathrm{GC}_{3} \mathrm{~s}\right)$ values was significant $(\mathrm{r}=-0.47, \mathrm{p}<$ 0.0001). Interestingly, this dimensional factor also significantly correlated with A content at synonymous variable third position $\left(\mathrm{A}_{3} \mathrm{~s}, \mathrm{r}=0.68, \mathrm{p}<0.0001\right)$ and $\mathrm{G}$ content at the same position $\left(\mathrm{G}_{3} \mathrm{~s}, \mathrm{r}=-0.71, \mathrm{p}<0.0001\right)$ (Figure 1). This means that the major factor shaping codon usage among these strains is an opposite trend between purines at third codon positions. Furthermore, this result is mainly due to the frequencies of the codons CGA (Arg) on one side of the distribution and GCG (Ala) and CGG (Arg) at the other side (see Additional file 1: Table S1). In other words, the differential usage of three low frequent codons ( $R S C U \leq 0.63$ ) is among the major factor shaping codon usage among these strains.

It has been suggested that dinucleotide biases can affect codon bias [19]. To study the possible effect of dinucleotide composition on codon usage of the H1N1pdm IAV strains, the relative abundances of the 16 dinucleotides in the ORFs of the 310 strains enrolled in these studies were established. The results of these analyses are shown in Table 2.

As it can be seen in the table, the occurrences of dinucleotides are not randomly distributed and no dinucleotides were present at the expected frequencies (Table 2). The relative abundance of CpG showed a strong deviation from the "normal range" (mean \pm S.D. $=0.319 \pm 0.0020$ ) and were markedly underrepresented. Interestingly, when the second 
Table 1 Codon usage in 2009 H1N1 pdm Influenza A Virus, displayed as RSCU ${ }^{a}$ values

\begin{tabular}{|c|c|c|c|c|c|c|c|c|c|c|c|c|c|}
\hline AA & Cod & $\mathrm{HC}$ & Swine & H1N1pdm & $\mathrm{H} 1 \mathrm{~N} 1^{b}$ & H3N2 & $\mathrm{AA}$ & Cod & $\mathrm{HC}$ & Swine & H1N1pdm & H1N1 & H3N2 \\
\hline \multirow[t]{2}{*}{ Phe } & UUU & 0.92 & 0.79 & 0.85 & 0.98 & 0.96 & Ser & UCU & 1.14 & 0.99 & 1.08 & 1.12 & 0.91 \\
\hline & UUC & 1.08 & 1.21 & 1.15 & 1.02 & 1.04 & & UCC & 1.32 & 1.50 & 0.74 & 0.87 & 0.97 \\
\hline \multirow[t]{6}{*}{ Leu } & UUA & 0.48 & 0.32 & 0.62 & 0.91 & 0.62 & & UCA & 0.90 & 0.73 & 1.57 & 1.62 & 1.34 \\
\hline & UUG & 0.78 & 0.67 & 1.00 & 1.27 & 1.30 & & UCG & 0.30 & 0.39 & 0.31 & 0.14 & 0.21 \\
\hline & CUU & 0.78 & 0.65 & 1.16 & 0.97 & 1.24 & Pro & CCU & 1.16 & 1.05 & 1.00 & 1.04 & 1.29 \\
\hline & CUC & 1.20 & 1.35 & 0.95 & 0.59 & 0.78 & & $\mathrm{CCC}$ & 1.28 & 1.46 & 0.80 & 0.72 & 0.84 \\
\hline & CUA & 0.42 & 0.33 & 1.20 & 1.00 & 0.96 & & $\mathrm{CCA}$ & 1.12 & 0.94 & 1.70 & 1.74 & 1.29 \\
\hline & CUG & 2.40 & 2.68 & 1.07 & 1.27 & 1.11 & & CCG & 0.44 & 0.56 & 0.50 & 0.49 & 0.58 \\
\hline \multirow[t]{3}{*}{ Ile } & $A \cup U$ & 1.08 & 0.91 & 1.07 & 1.07 & 1.03 & Thr & ACU & 1.00 & 0.83 & 1.01 & 1.11 & 1.28 \\
\hline & AUC & 1.41 & 1.67 & 0.77 & 0.78 & 0.89 & & ACC & 1.44 & 1.68 & 0.79 & 0.96 & 0.72 \\
\hline & AUA & 0.51 & 0.42 & 1.16 & 1.16 & 1.08 & & $A C A$ & 1.12 & 0.92 & 1.88 & 1.74 & 1.67 \\
\hline Met & AUG & 1.00 & 1.00 & 1.00 & 1.00 & 1.00 & & $A C G$ & 0.44 & 0.57 & 0.32 & 0.19 & 0.34 \\
\hline \multirow[t]{4}{*}{ Val } & GUU & 0.72 & 0.57 & 0.83 & 0.97 & 1.06 & Ala & GCU & 1.08 & 0.96 & 0.98 & 1.13 & 1.06 \\
\hline & GUC & 0.96 & 1.07 & 0.77 & 0.74 & 0.69 & & GCC & 1.60 & 1.80 & 0.87 & 0.87 & 0.93 \\
\hline & GUA & 0.48 & 0.34 & 1.12 & 1.07 & 1.02 & & GCA & 0.92 & 0.74 & 1.87 & 1.74 & 1.73 \\
\hline & GUG & 1.84 & 2.03 & 1.28 & 1.22 & 1.23 & & GCG & 0.44 & 0.50 & 0.27 & 0.26 & 0.28 \\
\hline \multirow[t]{2}{*}{ Tyr } & UAU & 0.88 & 0.73 & 1.04 & 1.09 & 1.13 & Cys & UGU & 0.92 & 0.79 & 0.88 & 1.09 & 0.79 \\
\hline & UAC & 1.12 & 1.27 & 0.96 & 0.91 & 0.87 & & UGC & 1.08 & 1.21 & 1.12 & 0.91 & 1.21 \\
\hline \multirow[t]{2}{*}{ TER } & UAA & $* *$ & $* *$ & $* *$ & $* *$ & $* *$ & TER & UGA & $* *$ & $* *$ & $* *$ & $* *$ & $* *$ \\
\hline & UAG & $* *$ & $* *$ & $* *$ & $* *$ & $* *$ & Trp & UGG & 1.00 & 1.00 & 1.00 & 1.00 & 1.00 \\
\hline \multirow[t]{2}{*}{ His } & CAU & 0.84 & 0.70 & 1.23 & 1.05 & 1.21 & Arg & CGU & 0.48 & 0.44 & 0.11 & 0.24 & 0.10 \\
\hline & CAC & 1.16 & 1.30 & 0.77 & 0.95 & 0.79 & & CGC & 1.08 & 1.31 & 0.33 & 0.18 & 0.24 \\
\hline \multirow[t]{2}{*}{ Gln } & CAA & 0.54 & 0.44 & 1.05 & 1.33 & 1.36 & & CGA & 0.66 & 0.60 & 0.63 & 0.41 & 0.43 \\
\hline & CAG & 1.46 & 1.56 & 0.95 & 0.67 & 0.64 & & CGG & 1.20 & 1.29 & 0.43 & 0.28 & 0.57 \\
\hline \multirow[t]{2}{*}{ Asn } & $\mathrm{AAU}$ & 0.94 & 0.79 & 1.15 & 1.20 & 1.15 & Ser & AGU & 0.90 & 0.77 & 1.14 & 1.15 & 0.95 \\
\hline & GAC & 1.08 & 1.21 & 0.95 & 0.80 & 0.85 & & AGC & 1.44 & 1.62 & 1.16 & 1.11 & 1.38 \\
\hline \multirow[t]{2}{*}{ Lys } & AAA & 0.86 & 0.76 & 1.10 & 1.27 & 1.39 & Arg & AGA & 1.26 & 1.12 & 2.89 & 3.08 & 2.84 \\
\hline & AAG & 1.14 & 1.24 & 0.90 & 0.73 & 0.61 & & AGG & 1.26 & 1.23 & 1.61 & 1.81 & 1.83 \\
\hline \multirow[t]{2}{*}{ Asp } & GAU & 0.92 & 0.80 & 1.05 & 1.13 & 1.08 & Gly & GGU & 0.64 & 0.57 & 0.57 & 0.60 & 0.69 \\
\hline & GAC & 1.08 & 1.20 & 0.95 & 0.87 & 0.92 & & GGC & 1.36 & 1.46 & 0.62 & 0.55 & 0.62 \\
\hline \multirow[t]{2}{*}{ Glu } & GAA & 0.84 & 0.72 & 1.20 & 1.15 & 1.14 & & GGA & 1.00 & 0.91 & 1.73 & 1.84 & 1.65 \\
\hline & GAG & 1.16 & 1.28 & 0.80 & 0.85 & 0.86 & & GGG & 1.00 & 1.05 & 1.08 & 1.01 & 1.04 \\
\hline
\end{tabular}

${ }^{a} R S C U$, relative synonymous codon usage; $A A$, amino acid; Cod, codons; HC, human cells; H1N1pdm, $2009 \mathrm{H} 1 \mathrm{~N} 1 \mathrm{pdm}$ Influenza A virus; $\mathrm{H} 1 \mathrm{~N} 1$ and $\mathrm{H} 3 \mathrm{~N} 2$, seasonal $\mathrm{H} 1 \mathrm{~N} 1$ and H3N2 Influenza A virus, respectively. Highly increased codons with respect to host cells $(\Delta \geq 0.30)$ are shown in bold. Codons containing de dinucleotide CG are shown in italics. ${ }^{b}$ RSCU codon usage of seasonal H1N1 and H3N2 according to Wong et al. (2010) [12].

dimensional factor (11.1\% of the total variability) was analyzed, we found that the position of each strain significantly correlated $(r=0.64, p<0.0001)$ with the respective usage of the dinucleotide CpG. Besides, although the global usage of this dinucleotide is very low, we found that the correlation is due to the differential usage of CGU (Arg) and CCG (Pro) codons, since these triplets display the most extreme values on the second dimensional factor (see Additional file 1: Table S1). Importantly, we also found that the third and the fourth dimensional factors of COA $(8.7 \%$ and $5.5 \%$ of the total variability, respectively), are again mainly linked to the low usage of codons containing the dinucleotide CpG, mainly at the positions 2 and 3. Moreover, among the 16 dinucleotides, 15 are highly correlated with the first dimensional factor value in COA (Table 2). These observations indicate that the composition of dinucleotides also plays a crucial role in the variation found in synonymous codon usage among H1N1pdm IAV ORFs.

To study the possibility of codon usage variation in the H1N1pdm IAV genomes enrolled in this study, the 

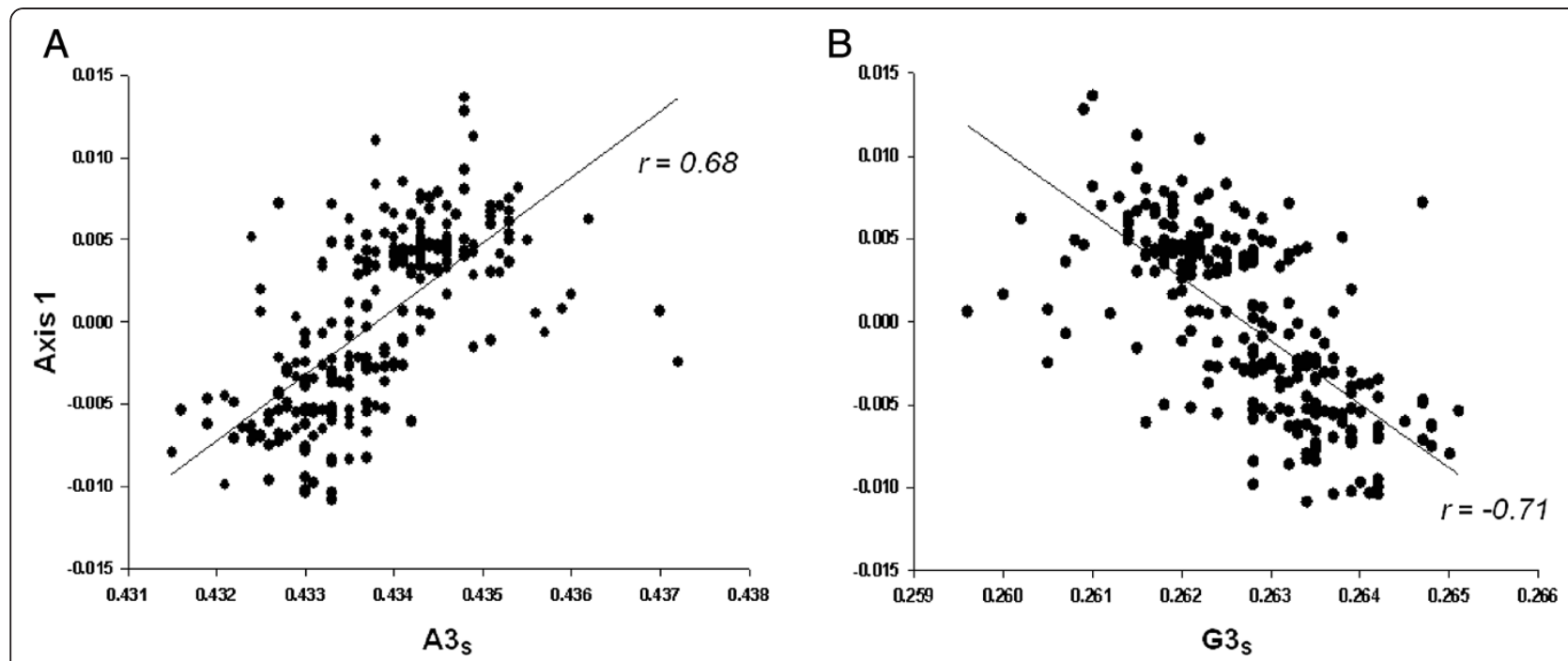

Figure 1 Association of purines at third codon positions with dimensional factor 1 generated by COA. In (A) and (B), the regression plots of the frequency of A3s and G3s versus the respective position of each strain in the first dimensional factor generated by the correspondence analysis on RSCU (COA-RSCU) are shown.

distribution of the 310 strains in the plane defined by the first two axes of COA was established. The results of these studies are shown in Figure 2.

Interestingly, the distribution of the H1N1pdm IAV strains in the plane defined by the first two major axes showed that the principal dimensional factor splits the strains at least three major groups: two of them discriminated by the first dimensional factor, while the third is revealed by the extreme low values on the second dimensional factor (Figure 2).

As the translation process represents a key step in the viral infection cycle, it is important to explore the strategies employed by the virus to harness the translation machinery of the cell host. Since variation at the third codon position makes possible the wobble interaction between that base and the first one of the anticodon [20], we wanted to gain further insight into the adaptation of H1N1pdm IAV strains to the respective host
tRNA pool context. For this reason, the codon usage of virus (H1N1pdm IAV) was plotted against the codon usage of host (human cells) and the nucleotide that occupy the first anticodon position (wobble position) of the corresponding codon was identified. The results of these studies are shown in Figure 3.

As it can be seen in the figure, codon usage of virus and host is uncorrelated. The viral preference toward AT rich genomes and the T-headed anticodons is clear (Figure 3). This is also in agreement with the consequence of a differential usage of $A 3_{s}$ and $G 3_{s}$ (see also Figure 1). Comparison of these findings with the compilation of tRNAs species in the human genome [21] reveals that the virus highly preferred T-headed anticodons are not particularly adapted to the host transfer tRNA pool (Table 3). Therefore, there is no obvious correlation between the number of human host isoacceptor tRNAs and codon usage of the IAV enrolled in these studies.

Table 2 Summary of correlation analysis between the dimensional factors (DF) in COA and sixteen dinucleotides frequencies in H1N1 pdm IAV ORFs

\begin{tabular}{|c|c|c|c|c|c|c|c|c|c|}
\hline & & UU & UC & UA & UG & $\mathrm{CU}$ & CC & $C A$ & CG \\
\hline$\overline{M e a n} \pm \mathrm{SD}^{a}$ & & $0.893 \pm 0.0054$ & $0.814 \pm 0.0050$ & $0.736 \pm 0.0009$ & $1.215 \pm 0.0009$ & $0.797 \pm 0.0056$ & $0.672 \pm 0.0033$ & $1.326 \pm 0.0042$ & $0.319 \pm 0.0020$ \\
\hline \multirow[t]{3}{*}{$\mathrm{DF} 1^{b}$} & r & 0.43277 & 0.30726 & 0.50328 & 0.49116 & 0.16033 & 0.40283 & 0.44451 & 0.47789 \\
\hline & $P$ & $<0.0001$ & $<0.0001$ & $<0.0001$ & $<0.0001$ & 0.0048 & $<0.0001$ & $<0.0001$ & $<0.0001$ \\
\hline & & $A U$ & $A C$ & AA & AG & GU & GC & GA & GG \\
\hline$\overline{M e a n} \pm \mathrm{SD}^{a}$ & & $1.281 \pm 0.0046$ & $0.926 \pm 0.0039$ & $1.804 \pm 0.0071$ & $1.327 \pm 0.0037$ & $0.682 \pm 0.0076$ & $0.703 \pm 0.0009$ & $1.472 \pm 0.0012$ & $1.040 \pm 0.0018$ \\
\hline \multirow[t]{2}{*}{$\mathrm{DF} 1^{b}$} & r & 0.44790 & 0.36540 & 0.61328 & 0.40489 & 0.08304 & 0.49579 & 0.48484 & 0.45555 \\
\hline & $P$ & $<0.0001$ & $<0.0001$ & $<0.0001$ & $<0.0001$ & 0.11880 & $<0.0001$ & $<0.0001$ & $<0.0001$ \\
\hline
\end{tabular}

${ }^{a}$ Mean values of $310 \mathrm{H} 1 \mathrm{~N} 1 \mathrm{pdm}$ IAV strains' relative dinucleotide ratios \pm standard deviation. ${ }^{b}$ Correlation analysis between the first dimensional factor in COA and the sixteen dinucleotides frequencies in H1N1 pdm IAV ORF's is shown. 


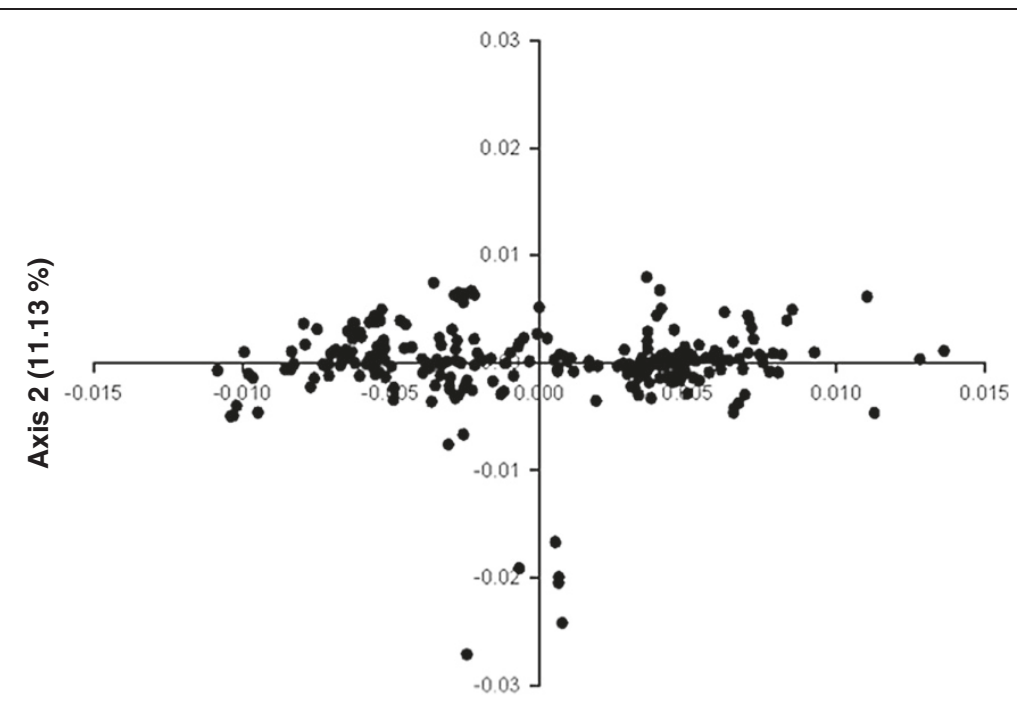

Axis $1(20.74 \%)$

Figure 2 Position of the $310 \mathrm{H} 1 \mathrm{~N} 1 \mathrm{pdm}$ IAV ORF's in the plane defined by the first two major axes generated by COA. The percentage of inertia of the first and second axes of COA is indicated for both axes between parentheses. The input values for COA were the RSCU values of each strain.

\section{Discussion}

As IAV relies on the host cell's machinery for its replication, codon usage bias could play a role in its adaptation to the host. The results of these studies revealed that codon usage in human IAV, including H1N1pdm, do not have the average codon usage pattern of their host's genes (see Table 1), in agreement with previous reports $[12,16]$.

Comparisons to previous results reported for other IAV such $\mathrm{H} 5 \mathrm{~N} 1$ (mean $\mathrm{ENC}=50.91$ ) $[16,22]$; or other RNA viruses like SARS (mean $E N C=48.99$ ) [23]; footand-mouth disease virus (mean $\mathrm{ENC}=51.42$ ) [24]; classical swine fever virus (mean $\mathrm{ENC}=51.7$ ) [19], Duck
Enteritis virus (mean ENC =52.17) [25], Encephalomyocarditis virus (mean $\mathrm{ENC}=54.86$ ) [26] or Theilovirus (mean ENC $=51.08$ ) [26], revealed that the ENC values found in this study for H1N1pdm IAV strains (mean ENC value of 52.5) are roughly similar to these previous findings, indicating that the overall extent of codon usage in these viruses are only slightly biased.

We have found a general link between codon usage bias and base composition, which is shown by the significant correlation of the position of each virus on the first dimensional factor of COA vs. the corresponding $\mathrm{GC}_{3} \mathrm{~S}$, together with the opposite trends in relation to purines at third codon position (Figure 1A and B). Taken

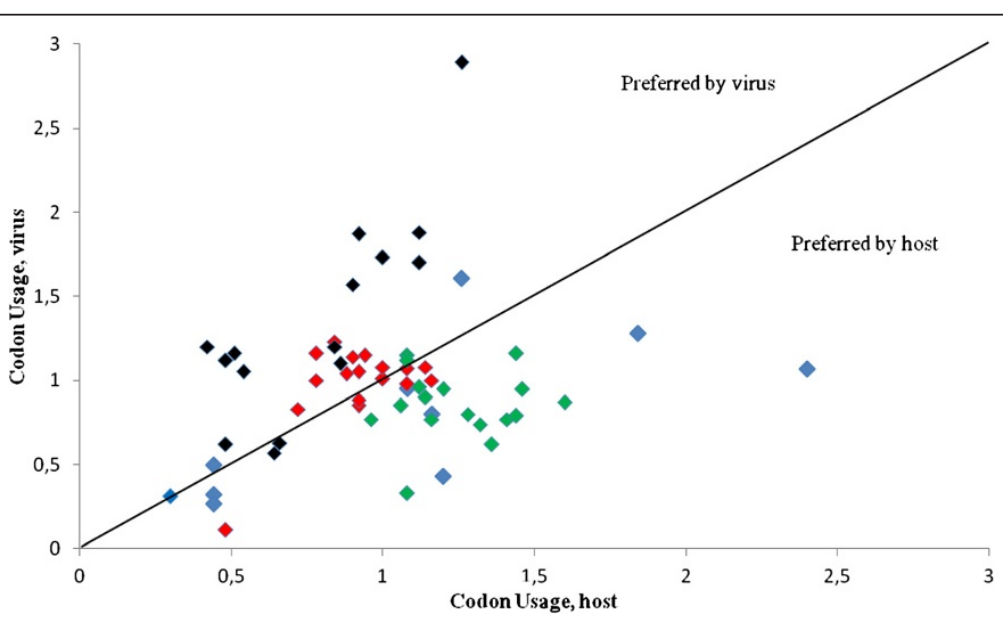

Figure 3 Codon usage of H1N1 pdm IAV plotted against the codon usage of human cells. Colors reflect the nucleotide that occupies the first anticodon position (wobble position) of the corresponding codon. A, C, G and T are indicated by red, blue, green and black diamonds, respectively. 
Table 3 Frequency of tRNA genes in human cells for highly biased codons in H1N1 pdm IAV*

\begin{tabular}{|c|c|c|c|}
\hline AA & Cod & Anticodon isotypes (tRNA count by anticodon) & Total tRNA anticodon count \\
\hline Ala & GCA & $\operatorname{UGC}(9), A G C(29), G G C(0), C G C(5)$ & 43 \\
\hline Arg & AGA \& AGG & $\mathrm{UCU}(6), \operatorname{CCU}(5), A C G(7), \mathrm{GCG}(0), \mathrm{CCG}(4), \mathrm{UCG}(6)$ & 28 \\
\hline Gln & CAA & $\operatorname{UUG}(\mathbf{1 1}), \operatorname{CUG}(21)$ & 32 \\
\hline Glu & GAA & $\operatorname{UUC}(\mathbf{1 3}), \mathrm{CUC}(13)$ & 26 \\
\hline Gly & GGA & $\operatorname{UCC}(9), \operatorname{GCC}(15), \operatorname{CCC}(7), A C C(0)$ & 31 \\
\hline His & CAU & $\operatorname{AUG}(0), G \cup G(11)$ & 11 \\
\hline \|le & AUA & $\mathbf{U A U}(\mathbf{5}), \operatorname{AAU}(14), \mathrm{GAU}(8)$ & 27 \\
\hline Leu & CUA & $\operatorname{UAG}(3), \operatorname{AAG}(12), \mathrm{CAG}(10), \mathrm{CAA}(7), \cup A A(7), \mathrm{GAG}(0)$ & 39 \\
\hline Pro & CCA & UGG(7), AGG(10), GGG(0), CGG(4) & 21 \\
\hline Ser & UCA & $\operatorname{UGA}(\mathbf{5}), \mathrm{AGA}(11), \mathrm{GGA}(0), \mathrm{CGA}(4), \mathrm{ACU}(0), \mathrm{GCU}(8)$ & 28 \\
\hline Thr & ACA & $\operatorname{UGU}(6), \mathrm{AGU}(10), \mathrm{GGU}(0), \mathrm{CGU}(6)$ & 22 \\
\hline Val & GUA & $\operatorname{UAC}(5), \operatorname{CAC}(16), \operatorname{AAC}(11), \operatorname{GAC}(0)$ & 32 \\
\hline
\end{tabular}

* Highly biased codons in H1N1 pdm IAV (as defined in Table 1) and their respective anticodons are shown in bold. AA, amino acid; Cod, codons.

together, our results indicate that the mutational bias is a very important trend in the evolution of H1N1pdm IAV genomes. However, this does not per se discards a role of other natural selection mechanisms acting in the IAV strains enrolled in these studies.

We have also found that CpG containing codons are sharply suppressed (see Table 1). This CpG deficiency was proposed to be related to the immunostimulatory properties of unmethylated $\mathrm{CpG}$, which are recognized by the innate immune system of the host as a pathogen signature [24,27]. This is triggered by the intracellular Pattern Recognition Receptor (PRR) Tool-like 9 (TLR9), which activates several immune response pathways [28]. It seems reasonable to suggest that exists among vertebrates a TLR9-like mechanism acting at the RNA level [29]. Interestingly, previous studies have shown that IAV strains originated from an avian reservoir and infecting human hosts since 1918 has been selected under strong pressure to reduce the frequency of $\mathrm{CpG}$ in its genome [30]. Marked CpG deficiency has been observed in several other RNA viruses [24,31-35], including H1N1pdm IAV $[12,30]$. Then, escaping from the host antiviral response may act as another selective pressure contributing to codon usage in H1N1pdm IAV strains [36].

The distribution of the $310 \mathrm{H} 1 \mathrm{~N} 1$ pdm IAV ORF's in the plane defined by the first two axes of COA shows the presence of at least three clusters of strains (Figure 2). Since species with a close genetic relationship always present a similar codon usage pattern [37] (see also Table 1), the results of these studies suggests that a dynamic process occurred in the H1N1pdm strains enrolled in these studies. This is reflected in the variation of codon usage observed among them (see Figure 2). These results suggest a balance of mutational bias and natural selection to shape codon usage in these strains, which allow the virus to explore and re-adapt its codon usage to different environments in a short period of time.

From the classical point of view, the preferred codons are recognized by the most abundant isoacceptors tRNAs, which implies the action of natural selection [38]. The results shown in Table 3 strongly suggest that this is not the case for H1N1pdm IAV strains. In other words, codon usage of these viruses does not seem to be adapted to the tRNA pool of the human cells but probably reflects the influence of mutational biases. Interestingly, this has been observed for some other RNA viruses, like HIV [39].

Understanding the mechanisms used by IAV to properly express its genes could suggest a novel point of intervention and drug targets. Reduced translation efficiency, particularly of structural genes that are needed for the formation of new particles, could affect viral success [40].

The results of this work suggest that synthetic attenuated virus engineering (SAVE) could play a role in creating new vaccines for IAV. By deoptimization of codon usage (replacing wild-type codons with codons and codon combinations whose sequences impair replication and/or expression), it might be possible to attenuate a virus [41]. Moreover, as the codon changes do not alter the protein sequence, the antigenicity should not differ from the wild-type virus. Besides, codon changes tend to have individually small fitness effects, so many nucleotide changes will be required to restore wild-type fitness, itself requiring $100 \mathrm{~s}$ or more generations [42-45]. This "death by a thousand cuts" strategy may provide an alternative method of attenuation [46]. Interestingly, it has been show that replacement of natural codons with synonymous triplets with increased frequencies of $\mathrm{CpG}$ gives rise to inactivation of Poliovirus infectivity [47]. Very recent studies revealed that this strategy can be applied to IAV [48]. 
Owing to known genome sequences, modern strategies of DNA synthesis have made it possible to recreate in principle all known viruses independent of natural templates [48]. Recoding of IAV to develop new vaccine candidates taking into account codon bias, base composition and adaptation to host tRNA by gene synthesis may provide important clues to elucidate virulence factors, identify targets for future drug intervention, and to develop new and appropriate vaccines [49].

\section{Methods}

\section{Sequences and dataset}

Sequences from H1N1pdm IAV strains, isolated from April to December of 2009, were obtained from The Influenza Virus Resource at the National Center for Biotechnological Information [50]. The data set comprised the complete genome sequences (eight segments) of 310 strains. For each strain the ORFs were concatenated $(\mathrm{PB} 2+\mathrm{PB} 1+\mathrm{PA}+\mathrm{HA}+\mathrm{NP}+\mathrm{NA}+\mathrm{MP}+\mathrm{NS})$ and aligned using the MUSCLE program [51]. The alignment is available upon request.

\section{Codon usage analysis}

Codon usage, base dinucleotide composition, $\mathrm{G}+\mathrm{C}$ at synonymous variable third position codons $\left(\mathrm{GC}_{3} \mathrm{~s}\right)$, the relative synonymous codon usage (RSCU) [14] and the effective number of codons (ENC) [17] were calculated using the program CodonW (written by John Peden and available at http://sourceforge.net/projects/codonw/) as implemented in the Mobile server (http://mobyle. pasteur.fr). Codon usage data of influenza viral hosts, human (Homo sapiens) and domestic swine (Sus scrofa) were obtained from the codon usage database (available at: http://www.kazusa.or.jp/codon) [52]. The frequencies of tRNAs in human cells were retrieved from the GtRNAdb database [21].

\section{Correspondence analysis(COA)}

$\mathrm{COA}$ is an ordination technique that identifies the major trends in the variation of the data and distributes genes along continuous axes in accordance with these trends. COA creates a series of orthogonal axes to identify trends that explain the data variation, with each subsequent dimensional factor explaining a decreasing amount of the variation [18]. Each ORF is represented as a 59-dimensional and each dimension is related to the RSCU value of each triplet (excluding AUG, UGG and stop codons). This was done using the CodonW program.

\section{Statistical analysis}

Correlation analysis was carried out using Spearman's rank correlation analysis method [53].

\section{Additional file}

Additional file 1: Table S1. Each codon included in the

correspondence analysis is represented by a row. Factor 1 and 2 columns

contain the coordinate of the codon on the respective generated axis.

\section{Competing interests}

The authors declare that they do not have competing interests.

\section{Authors' contributions}

JC conceived of the study, and participated in its design and coordination. NG and Al have made substantial contributions to the design of the study, acquisition of data and analysis. VC, MS, GM, GC, have been involved in revising the manuscript critically for important intellectual content. JC wrote the paper. HM helped to draft the manuscript and made substantial and fundamental contributions to the interpretation and discussion of the results found in this work. All authors read and approved the final manuscript.

\section{Acknowledgements}

We acknowledge support by International Atomic Energy Agency, through Research Contract No. 15792 and Comisión Sectorial de Investigación Científica (CSIC), Universidad de la República, Uruguay, through I + D Project "Variabilidad genetica y evolución viral de virus Influenza A en Uruguay". Authors acknowledge support by Agencia Nacional de Investigación e Innovación (ANII) through project PE_ALI_2009_1_1603 and PEDECIBA, Uruguay. We also thank the support of Fondo Clemente Estable, 2007_ 722 to HM.

\section{Author details}

'Laboratorio de Virología Molecular, Centro de Investigaciones Nucleares, Facultad de Ciencias, Universidad de la República, Iguá 4225, Montevideo 11400, Uruguay. ²Laboratorio de Organización y Evolución del Genoma, Instituto de Biología, Facultad de Ciencias, Universidad de la República, Iguá 4225, Montevideo 11400, Uruguay. ' Laboratorio de Evolución, Instituto de Biología, Facultad de Ciencias, Universidad de la República, Iguá 4225, Montevideo 11400, Uruguay. ${ }^{4}$ Unidad de Proteínas Recombinantes, Institut Pasteur de Montevideo, Mataojo 2020, Montevideo 11400, Uruguay. ${ }^{5}$ Unidad de Biofísica de Proteínas, Institut Pasteur de Montevideo, Mataojo 2020, Montevideo 11400, Uruguay.

Received: 23 February 2012 Accepted: 2 November 2012 Published: 8 November 2012

\section{References}

1. Neumann G, Brownlee GG, Fodor E, Kawaoka Y: Orthomyxovirus: replication, transcription, and polyadenylation. Curr Top Microbiol Immunol 2004, 283:121-143.

2. Ahn I, Son HS: Comparative study of the hemagglutinin and neuraminidase genes of Influenza A virus H3N2, H9N2 and H5N1 subtypes using bioinformatics techniques. Can J Microbiol 2007, 53:830-839.

3. Wolf YL, Viboud C, Holmes EC, Koonin EV, Lipman DJ: Long intervals of stasis punctuated by bursts of positive selection in the seasonal evolution of influenza A virus. Biol Direct 2006, 1:34.

4. Hillerman MR: Realities and enigmas of human viral influenza: pathogenesis, epidemiology and control. Vaccine 2002, 20:3068-3087.

5. De Jong JC, Rimmelzwaan GF, Fouchier RA, Osterhaus AD: Influenza virus: a master of metamorphosis. J Infection 2000, 40:218-228.

6. Ferguson NM, Galvani AP, Bush RM: Ecological and immunological determinants of influenza evolution. Nature 2003, 422:428-433.

7. World Health Organization: Pandemic (H1N1). Influenza-like illness in the United States and Mexico. 24 April 2009. 2009. Available: http://www.who.int/ csr/don/2009_04_24/en/index.html.

8. Centers for Disease Control and Prevention: Update: infections with a swine-origin influenza A (H1N1) virus - United States and other countries, April 28th, 2009. Morb Mortal Wkly Rep 2009, 58:431-433.

9. Smith GJD, Vijaykrishna D, Bahl J, Lycett SJ, Worobey M, Pybus OG, Ma SK, Cheung CL, Raghwani J, Bhatt S, Peiris JSM, Guan Y, Rambaut A: Origins and evolutionary genomics of the 2009 swine-origin H1N1 Influenza A epidemic. Nature 2009, 459:1122-1125. 
10. Gorman OT, Bean WJ, Kawaoka Y, Donatelli I, Guo YJ, Webster RG: Evolution of influenza A virus nucleocapsid genes: implications for the origins of H1N1 human and classical swine viruses. J Virol 1991, 65:3704-3714.

11. Stoletzki N, Eyre-Walker A: Synonymous codon usage in Escherichia coli: selection for translational accuracy. Mol Biol Evol 2007, 24:374-381.

12. Wong E, Smith DK, Rabadan R, Peiris M, Poon L: Codon usage bias and the evolution of Influenza A viruses. Codon usage biases of Influenza virus. Evol Biol 2010, 10:253.

13. Ikemura T: Correlation between the abundance of yeast transfer RNAs and the occurrence of the respective codons in protein genes. Differences in synonymous codon choice patterns of yeast and Escherichia coli with reference to the abundance of isoaccepting transfer RNAs. J Mol Biol 1982, 158:573-597.

14. Sharp PM, Li WH: An evolutionary perspective on synonymous codon usage in unicellular organisms. J Mol Evol 1986, 24:28-38.

15. Kryazhimskiy S, Bazykin GA, Dushoff J: Natural selection for nucleotide usage at synonymous and non-synonymous sites in the influenza $A$ genes. J Virol 2008, 82:4938-4945

16. Zhou T, Gu W, Ma J, Sun X, Lu Z: Analysis of synonymous codon usage in H5N1 virus and other influenza A viruses. Biosystems 2005, 81:77-86.

17. Comeron JM, Aguade M: An evaluation of measures of synonymous codon usage bias. J Mol Evol 1998, 47:268-274.

18. Greenacre M: Theory and applications of correspondence analysis. London: Academic; 1984

19. Tao P, Dai L, Luo M, Tang F, Tien P, Pan Z: Analysis of synonymous codon usage in classical swine fever virus. Virus Genes 2009, 38:104-112.

20. Crick FHC: Codon-anticodon pairing - Wobble hypothesis. J Mol Biol 1966, 19:548-555.

21. Chan PP, Lowe TM: GtRNAdb: a database of transfer RNA genes detected in genomic sequence. Nucleic Acids Res 2009, 37:D93-D97.

22. Li ZP, Ying DQ, Li P, Li F, Bo XC, Wang SQ: Analysis of synonymous codon usage bias in 09H1N1. Vir Sin 2010, 25:329-340.

23. Woo PCY, Wong BHL, Huang Y, Lau SKP, Yuen K: Cytosine deamination and selection of CpG suppressed clones are the two major independent biological forces that shape codon usage bias in Coronaviruses. Virology 2007, 369:431-442

24. Zhong J, Li Y, Zhao S, Liu S, Zhang Z: Mutation pressures shapes codon usage in the GC-rich genome of foot-and-mouth disease virus. Virus Genes 2007, 35:767-776.

25. Jia R, Cheng A, Wang M, Xin H, Guo Y, Zhu D, Qi X, Zhao L, Ge H, Chen X: Analysis of synonymous codon usage in the UL24 gene of duck enteritis virus. Virus Genes 2009, 38:96-103.

26. Liu WQ, Zhang J, Zhang YQ, Zhou JH, Chen HT, Ma LN, Ding YZ, Liu Y: Compare the differences of synonymous codon usage between the two species within cardiovirus. Virology J 2011, 8:325.

27. Shackelton LA, Parrish CR, Holmes EC: Evolutionary basis of codon usage and nucleotide composition bias in vertebrate DNA viruses. $J \mathrm{Mol}$ Evol 2006, 62:551-563.

28. Dorn A, Kippenberger S: Clinical application of CpG-, non-CpG, and antisense oligodeoxynucleotides as immunomodulators. Curr Opin $\mathrm{Mol}$ Ther 2008, 10:10-20.

29. Lobo FP, Mota BEF, Pena SDJ, Azevedo V, Macedo AM, Tauch A, Machado CR, Franco GR: Virus-host coevolution: common patterns of nucleotide motif usage in Flaviviridae and their hosts. PLoS One 2009, 4:6282

30. Greenbaum BD, Levine AJ, Bhanot G, Rabadan R: Patterns of evolution and host gene mimicry in influenza and other RNA viruses. PLoS Pathog 2008, 4:1000079.

31. Rabadan R, Levine AJ, Robins $\mathrm{H}$ : Comparison of avian and human influenza $A$ viruses reveals a mutational bias on the viral genomes. J Virol 2006, 80:11887-11891.

32. Rothberg PG, Wimmer E: Mononucleotide and dinucleotide frequencies and codon usage in poliovirus RNA. Nucleic Acids Res 1981, 9:6221-6229.

33. Karlin S, Doerfler W, Cardon LR: Why is CpG suppressed in the genomes of virtually all small eukaryotic viruses but not in those of large eukaryotic viruses? J Virol 1996, 68:2889-2897

34. Martínez-Gómez M, López-Tort F, Volotao-Ede M, Recarey R, Moratorio G, Musto $H$, Leite JP Cristina J: Analysis of human P[4]G2 rotavirus strains isolated in Brazil reveals codon usage bias and strong compositional constraints. Infec Genet Evol 2011, 11:580-586.
35. D'Andrea L, Pintó RM, Bosch A, Musto H, Cristina J: A detailed comparative analysis on the overall codon usage patterns in hepatitis $A$ virus. Virus Res 2011, 157:19-24.

36. Vetsigian K, Goldenfeld N: Genome rhetoric and the emergence of compositional bias. Proc Nat Acad Sci USA 2009, 106:215-220.

37. Sharp PM, Cowe E, Higgins DG, Shields DC, Wolfe KH, Wright F: Codon usage patterns in Escherichia coli, Bacillus subtilis, Saccharomyces cerevisiae, Schizosaccharomyces pombe, Drosophila melanogaster and Homo sapiens: a review of the considerable within-species diversity. Nucleic Acids Res 1988, 16:8207-8211.

38. Ikemura T: Codon usage and tRNA content in unicellular and multicellular organisms. Mol Biol Evol 1985, 2:13-34.

39. van Weringh A, Ragonnet-Cronin M, Pranckeviciene E, Pavon-Eternod M, Kleiman L, Xia X: HIV-1 modulates the tRNA pool to improve translation efficiency. Mol Biol and Evol 2011, 28:1827-1834.

40. Ngumbela KC, Ryan KP, Sivamurthy R, Brockman MA, Gandhi RT, Bhardwaj N, Kavanagh DG: Quantitative effect of suboptimal codon usage on translational efficiency of mRNA encoding HIV-1 gag in intact T cells. PLOS ONE 2008, 3:2356.

41. Coleman JR, Papamichail D, Skiena S, Futcher B, Wimmer E, Muller S: Virus attenuation by genome-scale changes in codon pair bias. Science 2008, 320:1784-1787.

42. Burns CC, Shaw J, Campagnoli R, Jorba J, Vincent A, Quay J, Kew O: Modulation of poliovirus replicative fitness in HeLa cells by deoptimization of synonymous codon usage in the capsid region. $J$ Virol 2006, 80:3259-3272.

43. Mueller S, Papamichail D, Coleman JR, Skiena S, Wimmer E: Reduction of the rate of poliovirus protein synthesis through large-scale codon deoptimization causes attenuation of viral virulence by lowering specific infectivity. J Virol 2006, 80:9687-9696.

44. Coleman JR, Papamichail D, Skiena S, Futcher B, Wimmer E, Mueller S: Virus attenuation by genome-scale changes in codon pair bias. Science 2008, 320:1784-1787.

45. Bull JJ, Molineux IJ, Wilke CO: Slow fitness recovery in a codon-modified viral genome. Mol Biol Evol 2012, doi:10.1093/molbev/mss119.

46. Mueller S, Coleman JR, Papamichail D, Ward CB, Nimnaual A, Futcher B, Skiena S, Wimmer E: Live attenuated Influenza virus vaccines by computer-aided rational design. Nature Biotech 2010, 28:723-726.

47. Burns CC, Campagnoli R, Shaw J, Vincent A, Jorba J, Kew O: Genetic inactivation of poliovirus infectivity by increasing the frequencies of CpG and UpA dinucleotides within and across synonymous capsid region codons. J Virol 2009, 83:9957-9969.

48. Wimmer E, Paul AV: Synthetic poliovirus and other designer viruses: what have we learned from them? Ann Rev Microbiol 2011, 65:583-609.

49. Wimmer E, Mueller S, Tumpey TM, Taubenberger JK: Synthetic viruses: a new opportunity to understand and prevent viral disease. Nature Biotech 2009, 27:1163

50. Bao Y, Bolotov D, Dernovoy B, Kiryutin L, Zaslavsky L, Tatusova T, Ostell J, Lipman D: The Influenza Virus Resource at the National Center for Biotechnology Information. J Virol 2008, 82:596-601.

51. Edgar RC: MUSCLE: a multiple sequence alignment method with reduced time and space complexity. BMC Bioinformatics 2004, 5:113.

52. Nakamura $Y$, Gojobori $T$, Ikemura T: Codon usage tabulated from international DNA sequence databases: status for the year 2000. Nucleic Acids Res 2000, 28:292.

53. Wessa P: Free Statistics Software, Office for Research Development and Education, version 1.1.23-r7. URL: http://www.wessa.net.

doi:10.1186/1743-422X-9-263

Cite this article as: Goñi et al:: Pandemic influenza A virus codon usage revisited: biases, adaptation and implications for vaccine strain development. Virology Journal 2012 9:263. 